\title{
A Novel Secretory Vesicle from Deer Antlerogenic Mesenchymal Stem Cell-Conditioned Media (DaMSC-CM) Promotes Tissue Regeneration
}

\author{
Minkoo Seo, ${ }^{1}$ Jin-Chul Kim, ${ }^{2}$ Hyung-Ki Kim, ${ }^{1}$ Eun wook Choi, ${ }^{1}$ Suyeong Jeong, \\ Ki Chang Nam $\left(\mathbb{1},{ }^{3}\right.$ and Mihue Jang $\mathbb{1}^{4}$ \\ ${ }^{1}$ Prostemics Research Institute, Seongdong-gu, Seoul, Republic of Korea \\ ${ }^{2}$ Natural Constituents of Research Center, Natural Products Research Institute, Korea Institute of Science and Technology (KIST), \\ Seoul, Republic of Korea \\ ${ }^{3}$ Department of Medical Engineering, Dongguk University College of Medicine, Seoul, Republic of Korea \\ ${ }^{4}$ Center for Theragnosis, Biomedical Research Institute, Korea Institute of Science and Technology (KIST), Seoul, Republic of Korea
}

Correspondence should be addressed to Ki Chang Nam; kichang.nam@gmail.com and Mihue Jang; mihue@kist.re.kr

Received 24 November 2017; Accepted 24 January 2018; Published 25 March 2018

Academic Editor: Jun Liu

Copyright (c) 2018 Minkoo Seo et al. This is an open access article distributed under the Creative Commons Attribution License, which permits unrestricted use, distribution, and reproduction in any medium, provided the original work is properly cited.

Multipotent stem cells have the capacity to generate terminally differentiated cell types of each lineage; thus, they have great therapeutic potential for a wide variety of diseases. The most widely available stem cells are derived from human tissues, and their use for therapeutic application is limited by their high cost and low productivity. Herein, we report that conditioned media of mesenchymal stem cells (MSCs) isolated from deer antlers enhanced tissue regeneration through paracrine action via a combination of secreted growth factors and cytokines. Notably, DaMSC-conditioned media (DaMSC-CM) enhanced hair regeneration by activating the Wnt signaling pathway. In addition, DaMSC-CM had regenerative potential in damaged skin tissue through induction of skin regeneration-related genes. Remarkably, we identified round vesicles derived from DaMSC-CM, with an average diameter of $\sim 120 \mathrm{~nm}$ that were associated with hair follicle formation, suggesting that secretory vesicles may act as paracrine mediators for modulation of local cellular responses. In addition, these secretory vesicles could regulate the expression of Wnt-3a, Wnt-10b, and lymphoid enhancer-binding factor-1 (LEF-1), which are related to tissue renewal. Thus, our findings demonstrate that the use of DaMSC-CM as a unique natural model for rapid and complete tissue regeneration has possible application for therapeutic development.

\section{Introduction}

Adult mesenchymal stem cells (MSCs) are self-renewing progenitor cells. Multipotent stem cells have the capacity to generate terminally differentiated cell types of each lineage which can fabricate specific tissues, including skin, bone, cartilage, and adipose tissue. To date, it has been widely accepted that stem cells play significant roles in tissue repair and regeneration [1-4]. Stem cell-based regenerative therapies have been successfully developed, indicating that stem cells have great merit as a source of regenerative medicine [5-7]. However, harnessing stem cells for this purpose requires a better understanding of the mechanisms by which existing tissues affect stem cell-mediated regenerative action.

In addition to the multipotent differentiation potential of MSCs, several paracrine factors of MSCs have been discovered that contribute to modulation of tissue regeneration $[8,9]$. MSCs can secrete bioactive molecules that act on cell migration, antioxidation, antiapoptosis, angiogenesis, and immunomodulation via regulation of local cellular responses [10-12]. Furthermore, several studies have demonstrated that injection of MSCs-CM also enhances tissue repair $[13,14]$. The release of paracrine factors such as growth factors and cytokines can affect the stem cell 
microenvironment in response to tissue injury, which can activate tissue survival, repair, and regeneration. Remarkably, recent studies reported that vesicles derived from stem cells may be key mediators of stem cell paracrine mechanisms [15-19]. For example, adipose mesenchymal stem cell(ASC-) derived extracellular vesicles can contribute to paracrine signaling [19]. Extracellular vesicles can be internalized by fibroblasts to promote cell migration, proliferation, and collagen synthesis. In addition, in vivo injection of extracellular vesicles into mice suffering from back wounds was shown to promote cutaneous wound healing by recruiting the vesicles to the damaged area. Thus, the multipotent potential of MSCs and paracrine mechanisms together affect repair and regeneration of damaged tissues.

Deer antlers are unique mammalian organs that undergo subsequent regrowth in response to seasonal changes throughout the animal's life and are thus a remarkably useful model of mammalian regeneration $[20,21]$. It has been suggested that the annual renewal of deer antlers involves a stem cell-based process [22]. Antler regeneration might be initiated by self-renewing multipotent stem cells residing in the pedicle periosteum (PP), a permanent protuberance on the frontal bone. PP-derived cells express several stem cell markers, such as CD9, OCT-4, Nanog, and STRO-1, and exhibit pluripotency due to their distinctive regenerative ability to differentiate along several mesenchymal lineages in vitro $[23,24]$. Nevertheless, the mechanism of regeneration in antlers remains unclear.

In this study, we demonstrated that conditioned media (CM) of cultured stem cells isolated from deer antlers play an important role in hair and skin regeneration by releasing paracrine factors that activate the Wnt signaling pathway. Furthermore, we discovered a novel mediator of paracrine actions in tissue regeneration, which indicates great potential for therapeutic application.

\section{Methods}

2.1. Isolation of Deer Antler-Derived MSCs (DaMSCs). Fresh red deer (Cervus elaphus) antlers were obtained under conditions approved by the local Animal Ethics Committee and in accordance with protocols approved by the Institute Animal Care. Antlers were collected from anesthetized four-year-old red deer stags during late spring from a local deer farm (Han-Lim-Won, Korea). The antler tip, composed of a velvet skin, perichondrium, mesenchyme, and chondroprogeniors, was taken using sterilized surgical instruments and then mesenchyme was obtained by slices of tissues. The obtained samples were digested with $0.075 \%$ collagenase type II (Sigma-Aldrich, USA) under gentle agitation for $45 \mathrm{~min}$ at $37^{\circ} \mathrm{C}$ and then centrifuged at $300 \mathrm{~g}$ for $10 \mathrm{~min}$ to obtain the stromal cell fraction. The pellets were filtered with a $70 \mathrm{~mm}$ nylon mesh and resuspended with phosphate-buffered saline (PBS). The solution was layered onto histopaque-1077 (Sigma-Aldrich, USA) and centrifuged at $840 \mathrm{~g}$ for $10 \mathrm{~min}$. The supernatant was discarded, and cell fraction was cultured overnight at $37^{\circ} \mathrm{C}$, in Dulbecco's Modified Eagle media (DMEM) supplemented with $10 \%$ fetal bovine serum (FBS), $100 \mathrm{U} / \mathrm{mL}$ of penicillin, and $100 \mathrm{mg} / \mathrm{mL}$ of streptomycin.
2.2. Immunocytofluorescence. Immunocytofluorescence labeling was conducted on cultured DaMSCs. To detect stem cell markers, anti-STRO-1 antibodies (RnD Systems, USA) were used in combination with fluorescein isothiocyanate(FITC-) conjugated anti-mouse IgM secondary antibodies (Molecular Probes, USA), and anti-CD90 antibodies (BD Biosciences, USA) were used in combination with Alexa Fluor 546-conjugated anti-mouse IgG secondary antibodies (Molecular Probes, USA). Primary antibodies were diluted $1: 50$ and secondary antibodies $1: 100$.

2.3. Protein Chip Assay. DaMSCs were incubated for 3 days in DMEM without FBS and then conditioned media of DaMSCs were harvested. After treatment of DaMSC-CM, a growth factor antibody array kit (Growth factor array C1, RayBio, USA) was accessed in according to the manufacturer's instructions. Normal media (DMEM) was treated as a negative control.

2.4. Cell Proliferation Assay. DaMSC-CM, ASC-CM, and normal media were used to treat three different types of cells including dermal papilla cells (DPCs), keratinocytes (HaCaTs) and human dermal fibroblasts (HDFs). At $48 \mathrm{~h}$ post incubation, a CCK-8 assay (Dojindo Molecular Technologies, USA) was conducted and cell proliferation was measured by absorbance at $450 \mathrm{~nm}$ using a microplate reader (Bio-Rad, USA).

2.5. Quantitative RT-PCR ( $q R T-P C R$ ) Analysis. After $72 \mathrm{~h}$ treatment with DMEM, ASC-CM, or DaMSC-CM, total RNA extracts from three different types of cells (DPCs, HaCaTs, and HDFs) were obtained using an RNeasy Mini Kit (Qiagen, Germany). Each $1 \mu \mathrm{g}$ of total RNA was reverse transcribed using the iScriptTM cDNA Synthesis Kit (Invitrogen, Korea), and $50 \mathrm{ng}$ of cDNA for each sample was used for qRT-PCR analysis (StepOne Real-Time PCR system, ABI) with the specific primers listed in Supplementary Table S1.

2.6. In Vitro Wound Healing Assay. For the measurement of cell migration, HDFs kept in serum-free media were placed onto a $60 \mathrm{~mm}$ dish to create a confluent monolayer and then wounding was generated with a plastic micropipette tip. After washing, the medium was replaced with control media or DaMSC-CM in a time-dependent manner. Photographs of wound spaces were taken by phase-contrast microscopy.

2.7. Isolation of Extracellular Vesicles (EVs) from DaMSC$C M$. To isolate vesicles secreted from DaMSCs, conditioned media were collected. After collection of conditioned media, cell debris and large membrane particles were removed by sequential centrifugation at $500 \mathrm{~g}$ for $30 \mathrm{~min}$ and then $10,000 \mathrm{~g}$ for $30 \mathrm{~min}$. Vesicles were collected after ultracentrifugation at $100,000 \mathrm{~g}$ for $1 \mathrm{~h}$ and then resuspended in PBS for further experiments.

2.8. Characterization of EVs Isolated from DaMSC-CM. To analyze the size and morphology of EVs, nanoparticle tracking analysis (NTA) and transmission electron microscopy (TEM) analysis were performed, respectively. For 


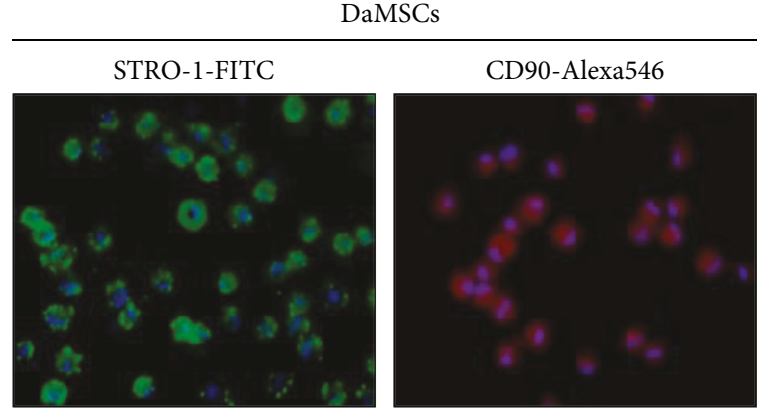

(a)

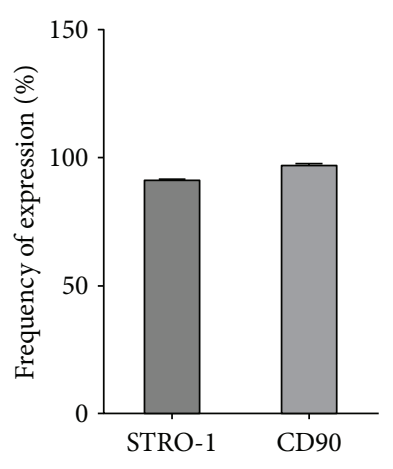

(b)

Figure 1: Immunolocalization of mesenchymal stem cell makers in deer antler-derived mesenchymal stem cells (DaMSCs). Immunocytochemistry staining was conducted on DaMSCs with primary antibodies directed against STRO-1 (a, left, green), and CD90 (a, right, red) and stained by FITC- and Alexa 546-conjugated secondary antibodies, respectively. Nuclei were visualized with DAPI (blue). (b) Graph indicates quantification of protein expression. Data represent the mean \pm s.d. $(n=3)$.

Control

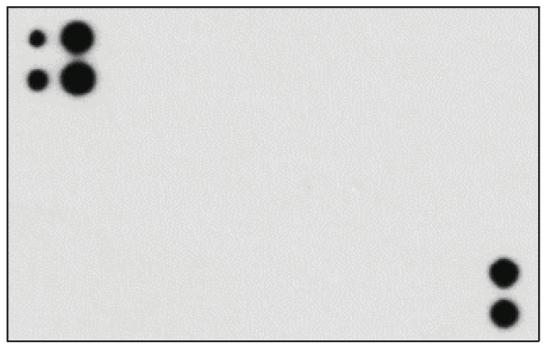

DaMSCs-CM

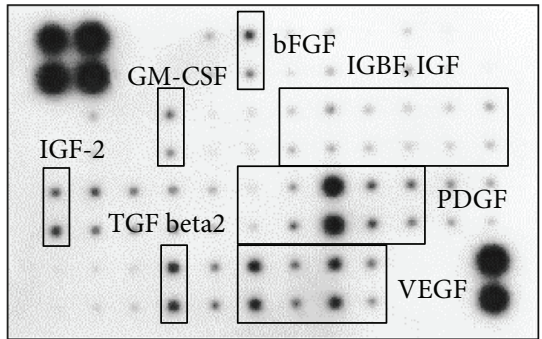

FIGURE 2: Growth factor secretion profiles of DaMSC-conditioned media (DaMSC-CM). A human growth factor antibody array was used to detect paracrine factors in DaMSC-CM. DMEM was used as a negative control.

measurement of particle concentrations, a NanoSight LM 10 instrument (NanoSight Ltd, Amesbury, UK) was used. For TEM images, vesicles fixed in $2 \%$ paraformaldehyde were transferred onto Formvar-carbon-coated electron microscopy grids. Grids were washed with PBS several times and covered with one drop of $1 \%$ glutaraldehyde for $5 \mathrm{~min}$. After washing with distilled water, grids were negatively stained with $2 \%$ uranyl acetate and analyzed with a transmission electron microscope (JEM-1011, Germany).

\section{Results}

3.1. Identification of DaMSCs. To verify the presence of MSCs, red deer antler-derived MSCs were isolated using a sequential centrifugation approach. Immunohistochemistry assays were used to detect stem cell markers including STRO-1 and CD90 in DaMSCs (Figure 1). STRO-1-positive or CD90-positive MSCs were visualized using fluorescence microscopy (Figure 1(a)) and quantified by normalization with DAPI-stained nuclei (Figure 1(b)). In three randomly selected regions of a culture dish, $>90 \%$ of observed cells were STRO-1-positive and $>95 \%$ were CD90-positive.

\subsection{Detection of Active Biomolecules from Conditioned Media} of DaMSCs. Recently, active biomolecules secreted from stem cells have been considered to have benefits in tissue regeneration because secreted molecules such as cytokines, growth factors, and extracellular matrix (ECM) molecules can contribute to the host tissue's microenvironment. Thus, we tested the paracrine effects of stem cells using a growth factor chip array. After treating media with DaMSCs, 26 secreting growth factors were detected in the conditioned media (Figure 2 and Supplementary Table S2). Normal media was used for a negative control for the chip array. Among growth factors, basic fibroblast growth factor (bFGF), insulin-like growth factor binding protein (IGFBP), insulin-like growth factors (IGFs), granulocyte macrophage colony-stimulating factor (GM-CSF), platelet-derived growth factors (PDGFs), vascular endothelial growth factors (VEGFs), and transforming growth factor- $\beta 2$ (TGF- $\beta 2$ ) were discovered. In particular, PDGF, VEGF, and TGF- $\beta 2$ were significantly released from DaMSCs.

\subsection{Effects of DaMSC-CM on PDC Proliferation. Mesen-} chyme-derived dermal papilla cells (DPCs), located at the bottom of the hair follicle, play important roles in regulating hair follicle generation $[25,26]$. Hair follicle development is deeply associated with signaling between epithelial keratinocytes and DPCs [27]. In particular, Wnt/ $\beta$-catenin signaling is required for the hair-inducing activity of dermal papillae, which is crucial for the proliferation of DPCs [28]. Thus, we first tested whether DaMSC-CM can induce DPC 


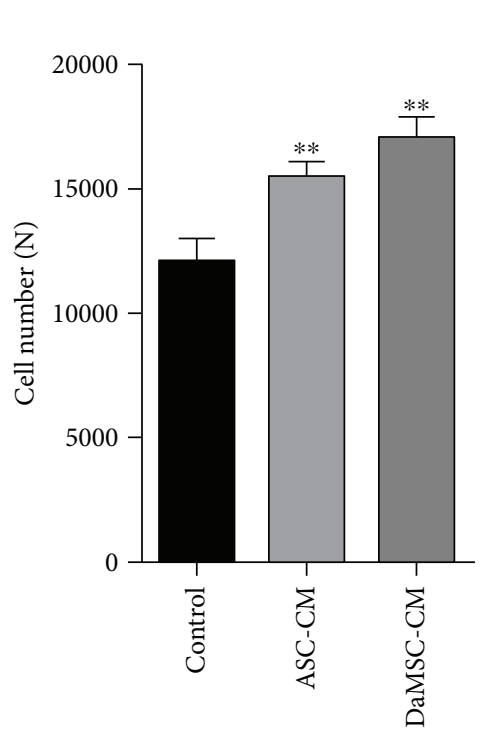

(a)
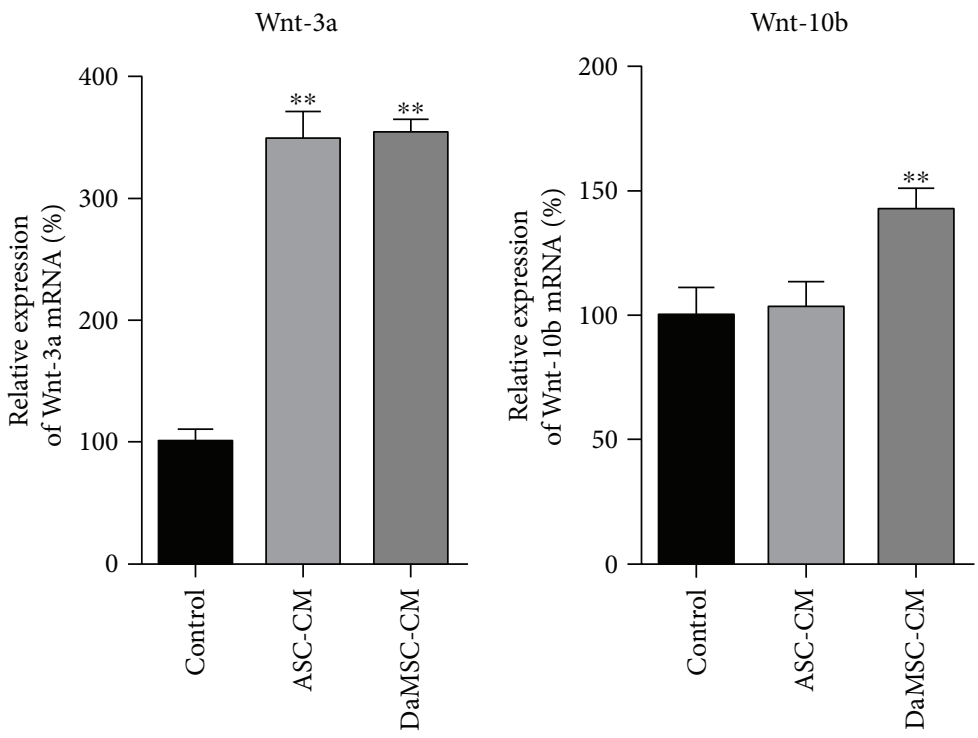

(b)

FIGURE 3: Effects of hair cell development on treatment with DaMSC-CM. (a) Proliferation of dermal papilla cells (DPCs) treated with control, adipose tissue-derived stem cell conditioned media (ASC-CM), or DaMSC-CM for $48 \mathrm{~h}$. (b) Activation of Wnt signaling in DPCs treated with control, ASC-CM, and DaMSC-CM. qRT-PCR analysis exhibits relative expression of Wnt-3a mRNA (b, left) and Wnt-10b mRNA (b, right), normalized to expression of GAPDH mRNA. Data represent the mean \pm s.d. $(n=3) .{ }^{* *} P<0.001$ by one-way analysis of variance with Tukey's multiple comparison tests, as compared with the control.

proliferation (Figure 3(a)). DaMSC-CM was used to treat DPCs for $48 \mathrm{~h}$ and then cell proliferation was evaluated using a CCK-8 assay. We also used adipose tissue-derived stem cell-conditioned media (ASC-CM) for comparison. Interestingly, DaMSC-CM promoted significantly more cell growth than ASC-CM. Additionally, DaMSC-CM showed almost 1.4-fold increase in cell number compared to normal media. Next, Wnt signaling seems to play important roles in the mechanisms of tissue repair and regeneration in both fetal and adult wounds $[29,30]$. The Wnt pathway also regulates cell proliferation in wound healing. In particular, skin wounds express various Wnt proteins with transcripts of Wnt-1, -3a, -4, -5a, and -10b [31]. In addition, hair follicle regeneration and MSC activation can be induced via activation of $\mathrm{Wnt} / \beta$-catenin signaling pathway regarding to Wnt-3a and Wnt-10b [32]. Thus, Wnt proteins such as Wnt-3, Wnt-10a, and Wnt-10b play a key role in hair follicle initiation, morphogenesis, and development [33]. To study the effect of Wnt signaling on hair growth, expression of Wnt-3a and Wnt-10b was analyzed using quantitative RT-PCR (Figures 3(b) and 3(c)).

Treatment with DaMSC-CM led to a 3.2-fold increase in Wnt-3a mRNA and 1.5-fold increase in Wnt-10b mRNA compared to normal media. Therefore, DaMSC$\mathrm{CM}$ promotes activation of Wnt signaling pathways for hair regeneration.

3.4. Effects of DaMSC-CM on Skin Regeneration. Several studies have revealed that stem cells closely contribute to wound repair after damage, resulting in regeneration of damaged skin $[34,35]$. Thus, we tested the effects of DaMSC-CM on wound healing. First, we investigated the proliferation of skin-related cells including keratinocytes (HaCaTs) and human dermal fibroblasts (HDFs) upon treatment with DaMSCs (Figure 4(a)). Compared to normal media, DaMSC-CM enhanced proliferation of both types of skin cells. In addition, we investigated the expression of skin regeneration-related genes after treatment with DaMSCCM (Figure 4(b)). Remarkably, DaMSC-CM treatment led to high levels of collagen type 1 and bFGF mRNA compared with normal media. Furthermore, to test the effects of DaMSC-CM on cell migration, a time-dependent in vitro wound healing assay was carried out (Figure 4(c)). Similar to our findings of cell proliferation, we observed enhanced migration of HDFs after artificial scratches of a confluent cell monolayer when treated with DaMSC-CM, especially at $72 \mathrm{~h}$ posttreatment, when the migration rate of cells treated with DaMSC-CM was approximately 75\%. Thus, DaMSC-CM also has regenerative effects on skin wounds.

3.5. Isolation of Secretory Vesicles from DaMSC-CM as Mediator of Paracrine Actions. Recent studies reported that EVs from stem cells may contribute to paracrine signaling $[19,36,37]$; therefore, we tried to purify secretory vesicles from DaMSC-CM (Figure 5). Surprisingly, many vesicles were detected when isolated from DaMSC-CM using an ultracentrifugation approach. NTA analysis obtained EVs with a size distribution of average $119.9 \mathrm{~nm}$ (Figure 5(a)), and TEM images exhibited nano-sized vesicles with lipid bilayers (Figure 5(b)). Next, to investigate the potential function of EVs isolated from DaMSC-CM on tissue regeneration, gene expression related to Wnt signaling pathways and hair follicle formation were evaluated after treatment of DaMSC-CM with EVs (Figure 6). Interestingly, EVs alone 


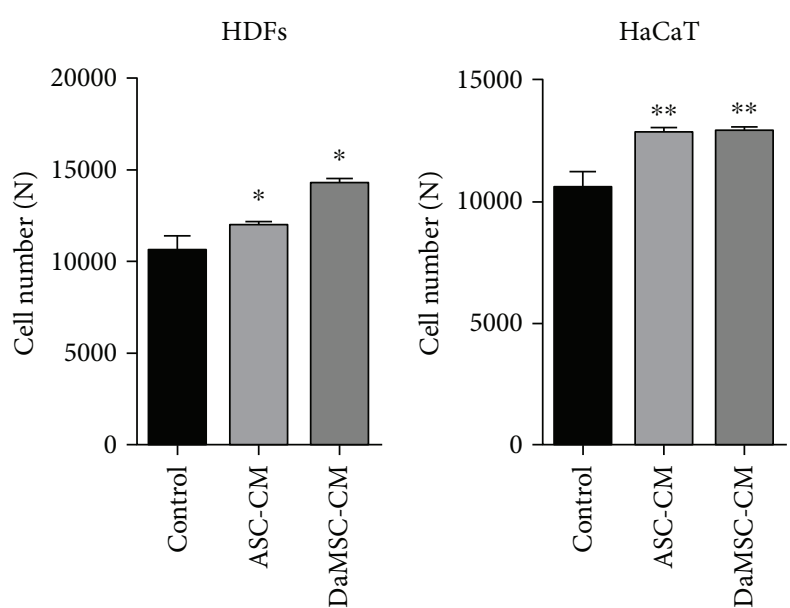

(a)
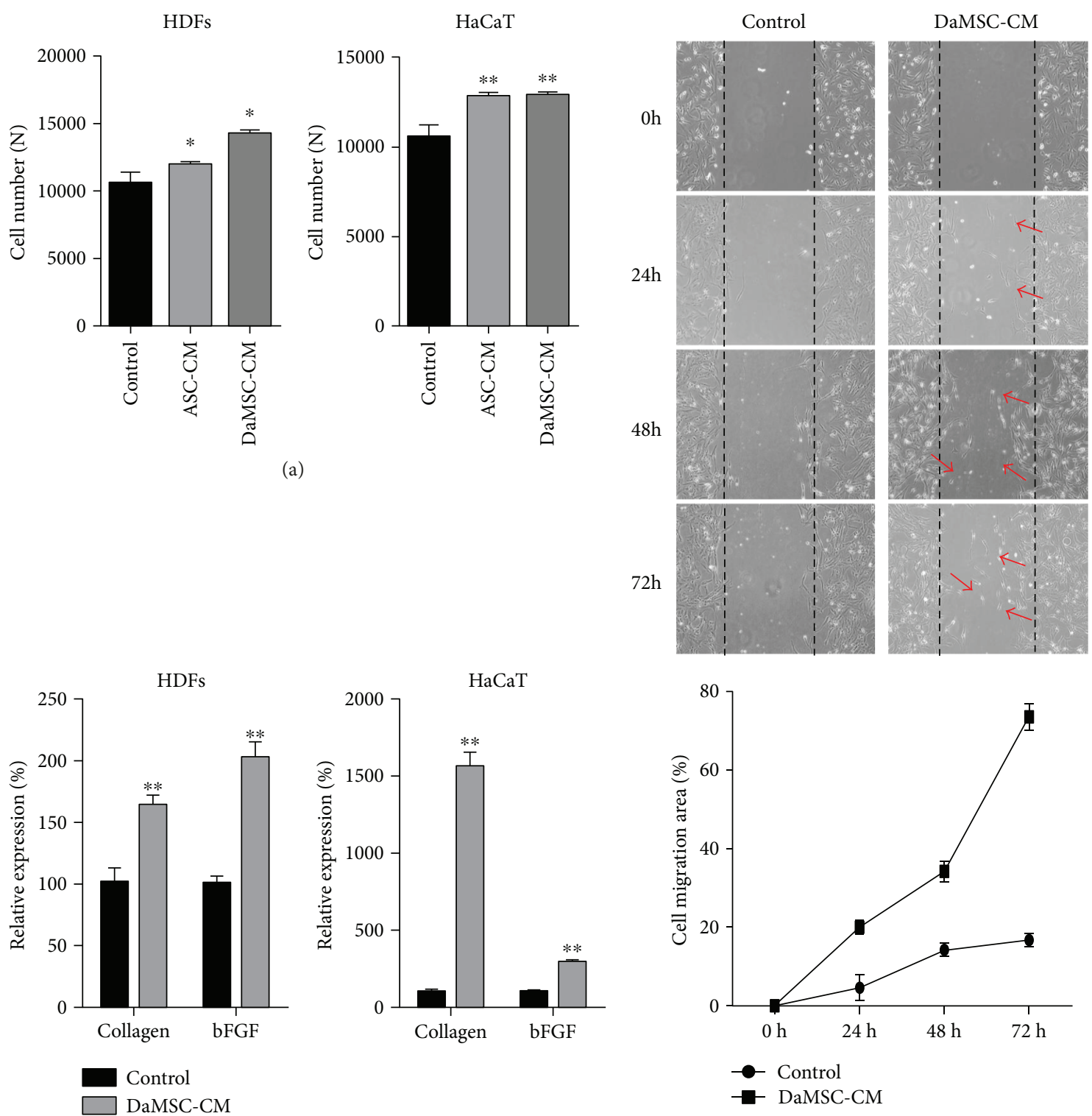

(b)

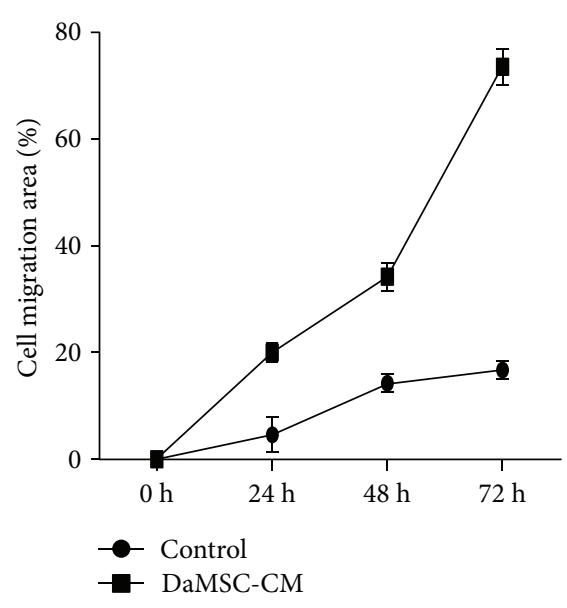

(c)

FIgURE 4: Effects of skin regeneration on treatment with DaMSC-CM. (a) Proliferation of human dermal fibroblasts (HDFs) and keratinocytes (HaCaTs) under treatment of three different types of media for $48 \mathrm{~h}$. (b) qRT-PCR analysis showing relative expression of collagen type I mRNA (b, left) and basic fibroblast growth factor (bFGF) mRNA (b, right), normalized to expression of GAPDH mRNA. (c) In vitro wound healing assay exhibiting HDF migration upon treatment with DaMSC-CM in a time-dependent manner. Red arrows indicate cell migration. Black lines represent wound spaces created with plastic micropipette tips. Data represent the mean \pm s.d. $(n=3)$. ${ }^{*} P<0.01,{ }^{* *} P<0.001$ by one-way analysis of variance with Tukey's multiple comparison tests, as compared with the control.

highly increased mRNA levels of Wnt-3a and Wnt-10b $\sim 8$-fold and $\sim 4$-fold, respectively. In contrast, our previous data showed that DaMSC-CM induced only 3.2-fold and 1.5-fold increases in the mRNA levels of Wnt-3a and Wnt-10b (Figure 3(b)). We assumed that EVs isolated from DaMSCs are highly enriched with growth factors and other factors. In addition, the level of LEF1 mRNA, which is a major endpoint mediator of the Wnt signaling pathway [38], was significantly increased under the treatment with EVs 5.6-fold (Figure 6). Thus, DaMSCs secrete extracellular vesicles that can act as mediators of paracrine actions.

\section{Discussion}

Stem cells have great potential to differentiate and regenerate into all types of cells and are thus unique cells in the body. Stem cell-based therapies have been conducted in auto- and allogenic transplantation for reconstruction of tissues. Many molecular components contribute to regenerative processes 


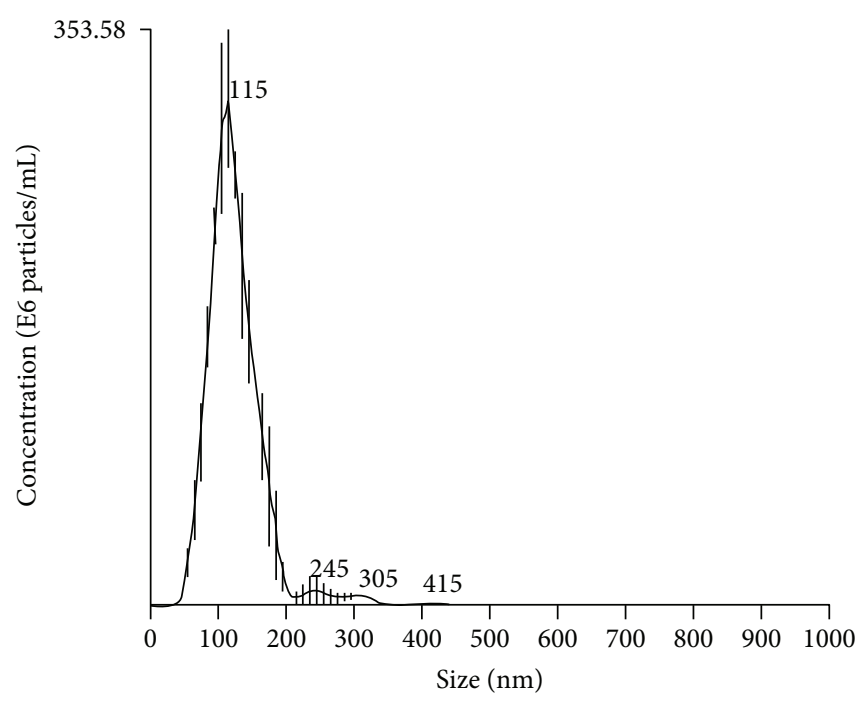

(a)

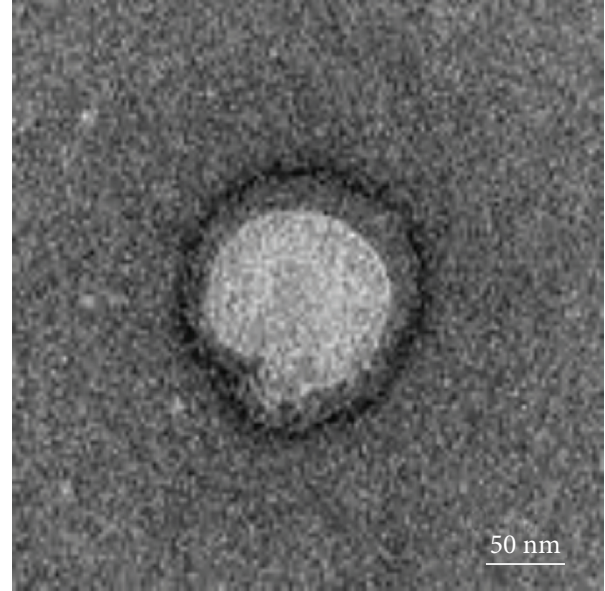

(b)

FIgURE 5: Characterization of extracellular vesicles (EVs) derived from DaMSCs. (a) Nanoparticle tracking analysis (NTA) showed a size distribution of EVs with an average of $119.9 \mathrm{~nm}$. (b) Transmission electron microscopic (TEM) images revealed the morphology of the EVs with a membrane bilayer. Scale bar indicates $100 \mathrm{~nm}$. Particle number: $2.29 \times 10^{15}$ particles $/ \mathrm{mL}$.
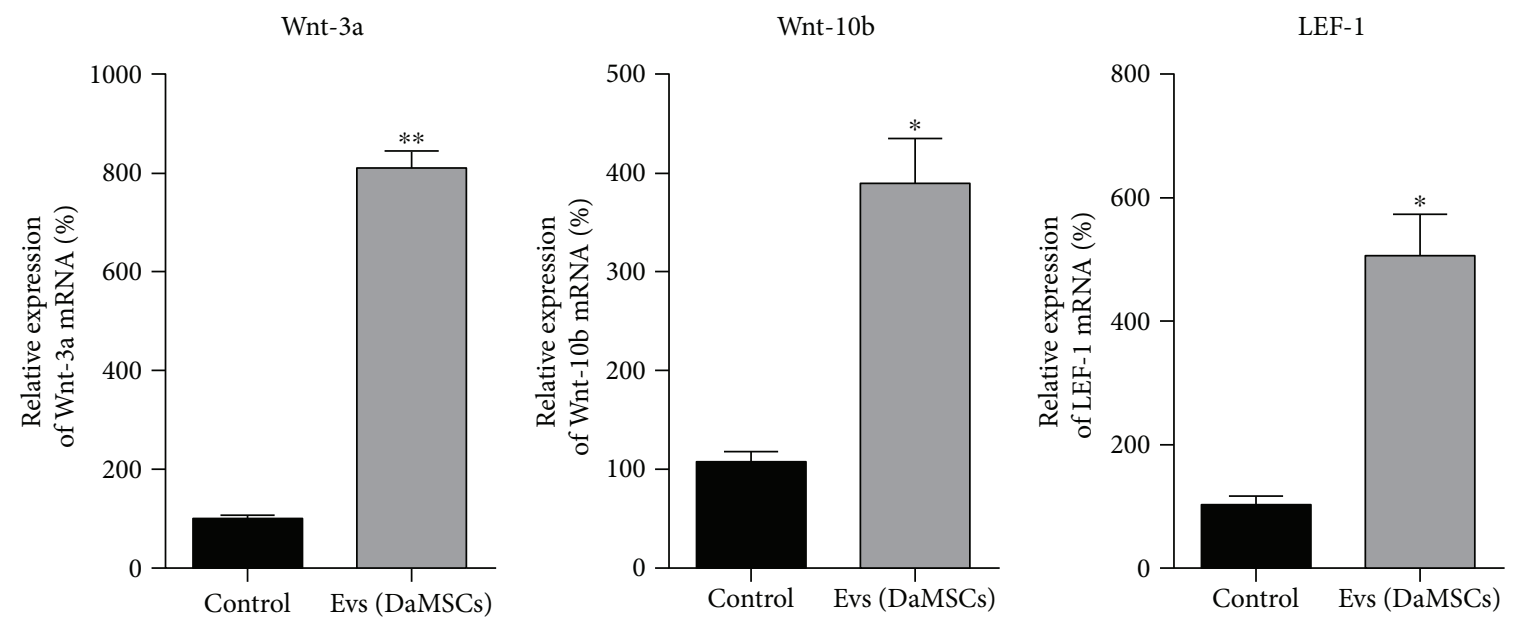

FIGURE 6: Activation of skin regeneration-related genes upon treatment with EVs. qRT-PCR analysis was conducted after treatment of DPCs with EVs alone. DMEM treatment was used as a negative control. mRNA expression of Wnt-3a, Wnt-10b, and lymphoid enhancer-binding factor-1 (LEF-1) was normalized to the expression of GAPDH mRNA. Data represent the mean \pm s.d. $(n=3)$. ${ }^{*} P<0.01,{ }^{* *} P<0.001$ by oneway analysis of variance with Tukey's multiple comparison tests, as compared with the control.

and have influences on engraftment [39, 40]. Stem cells with the ability for homing and self-renewal play crucial roles in mechanisms of tissue regeneration, but paracrine actions are also essential for restoration of tissues. In addition to their multipotent differentiation potential, stem cells can release cytokines, chemokines, and growth factors after injury. These paracrine factors can influence adjacent cells and their microenvironments. Interestingly, paracrine mediators seem to be expressed in response to injury. It had previously been reported that intravenous and intracoronary injection of MSCs-CM significantly restored ventricular performance in an injury model [41]. Notably, nanovesicles in CM were discovered with a range of $100-220 \mathrm{~nm}$ in diameter, suggesting that paracrine signaling may be affected by EVs acting as mediators between cells. Several approaches, including proteomics, chip arrays, microarrays, and other high-throughput analyses, have been performed to identify the content of vesicles [17]. Recently, many studies have discovered the function of MSC-derived exosomes on therapeutic effects [42]. Secretary vesicles including exosomes and microvesicles are involved in direct or indirect cell-to-cell communication, thereby altering tissue environment and metabolism. These vesicles carry diverse cytokines and growth factors, lipids, small RNAs, and so on, which can influence tissue responses 
to injury, disease, and infection. Surprisingly, MSCs and MSC-derived exosomes exhibit similar therapeutic effects in sparing tissue or promoting regeneration from injury sites $[43,44]$. Thus, MSC-derived exosomes have great merit in cell-free therapeutics.

In our study, we also revealed that DaMSCs released high levels of soluble factors that may induce activation of canonical Wnt/ $\beta$-catenin signaling. The EVs that we found in this study may also influence tissue regeneration as paracrine mediators. Accordingly, it has been proposed that Wnts are secreted on exosomes and contribute to tissue injury repair $[45,46]$. Thus, our investigation of DaMSC-derived EVs acting in tissue regeneration gives rise to a new aspect of regenerative medicine in which EVs may be a unique material for rapid and complete tissue regeneration.

\section{Conclusions}

In this study, we demonstrated that DaMSC-CM can significantly promote tissue regeneration such as hair follicle formation and skin wound healing, which are both associated with Wnt signaling pathways. DaMSCs can release bioactive molecules including PDGF, VEGF, and TGF- $\beta 2$ as paracrine factors for cell-to-cell communication. Furthermore, we identified nano-sized vesicles derived from DaMSC-CM and investigated their ability to promote hair follicle formation via Wnt signaling. These results suggested that secretory vesicles act as paracrine mediators for regulation of cellular responses. Thus, our findings indicate great potential for application in regenerative medicine.

\section{Conflicts of Interest}

The authors declare that they have no conflicts of interest.

\section{Acknowledgments}

This study was supported by the KIST Institutional Program (Project no. 2E27950).

\section{Supplementary Materials}

Table S1: a list of primers for qRT-PCR analysis. The relative expression of each mRNA was analyzed using qRT-PCR and normalized by the expression of GAPDH mRNA. Table S2: a list of secreted growth factors. The paracrine effects of stem cells using a growth factor chip array were tested. (Supplementary Materials)

\section{References}

[1] G. F. Curley, B. Ansari, M. Hayes et al., "Effects of intratracheal mesenchymal stromal cell therapy during recovery and resolution after ventilator-induced lung injury," Anesthesiology, vol. 118, no. 4, pp. 924-932, 2013.

[2] H. Reinshagen, C. Auw-Haedrich, R. V. Sorg et al., "Corneal surface reconstruction using adult mesenchymal stem cells in experimental limbal stem cell deficiency in rabbits," Acta Ophthalmologica, vol. 89, no. 8, pp. 741-748, 2011.
[3] D. Matsuse, M. Kitada, M. Kohama et al., "Human umbilical cord-derived mesenchymal stromal cells differentiate into functional Schwann cells that sustain peripheral nerve regeneration," Journal of Neuropathology and Experimental Neurology, vol. 69, no. 9, pp. 973-985, 2010.

[4] K. H. Schuleri, G. S. Feigenbaum, M. Centola et al., "Autologous mesenchymal stem cells produce reverse remodelling in chronic ischaemic cardiomyopathy," European Heart Journal, vol. 30, no. 22, pp. 2722-2732, 2009.

[5] A. B. Mathiasen, A. A. Qayyum, E. Jorgensen et al., "Bone marrow-derived mesenchymal stromal cell treatment in patients with severe ischaemic heart failure: a randomized placebo-controlled trial (MSC-HF trial)," European Heart Journal, vol. 36, no. 27, pp. 1744-1753, 2015.

[6] O. Y. Bang, "Clinical trials of adult stem cell therapy in patients with ischemic stroke," Journal of Clinical Neurology, vol. 12, no. 1, pp. 14-20, 2016.

[7] K. W. Oh, C. Moon, H. Y. Kim et al., "Phase I trial of repeated intrathecal autologous bone marrow-derived mesenchymal stromal cells in amyotrophic lateral sclerosis," Stem Cells Translational Medicine, vol. 4, no. 6, pp. 590-597, 2015.

[8] P. R. Baraniak and T. C. McDevitt, "Stem cell paracrine actions and tissue regeneration," Regenerative Medicine, vol. 5, no. 1, pp. 121-143, 2010.

[9] D. F. Anthony and P. G. Shiels, "Exploiting paracrine mechanisms of tissue regeneration to repair damaged organs," Transplantation Research, vol. 2, no. 1, p. 10, 2013.

[10] H. R. Hofer and R. S. Tuan, "Secreted trophic factors of mesenchymal stem cells support neurovascular and musculoskeletal therapies," Stem Cell Research \& Therapy, vol. 7, no. 1, p. 131, 2016.

[11] L. Biancone, S. Bruno, M. C. Deregibus, C. Tetta, and G. Camussi, "Therapeutic potential of mesenchymal stem cell-derived microvesicles," Nephrology, Dialysis, Transplantation, vol. 27, no. 8, pp. 3037-3042, 2012.

[12] Y. Zhang, S. Liao, M. Yang et al., "Improved cell survival and paracrine capacity of human embryonic stem cell-derived mesenchymal stem cells promote therapeutic potential for pulmonary arterial hypertension," Cell Transplantation, vol. 21, no. 10, pp. 2225-2239, 2012.

[13] M. Gnecchi, Z. Zhang, A. Ni, and V. J. Dzau, "Paracrine mechanisms in adult stem cell signaling and therapy," Circulation Research, vol. 103, no. 11, pp. 1204-1219, 2008.

[14] L. Chen, E. E. Tredget, P. Y. Wu, and Y. Wu, "Paracrine factors of mesenchymal stem cells recruit macrophages and endothelial lineage cells and enhance wound healing," PLoS One, vol. 3, no. 4, article e1886, 2008.

[15] X. Liang, Y. Ding, Y. Zhang, H. F. Tse, and Q. Lian, “Paracrine mechanisms of mesenchymal stem cell-based therapy: current status and perspectives," Cell Transplantation, vol. 23, no. 9, pp. 1045-1059, 2014.

[16] S. R. Baglio, D. M. Pegtel, and N. Baldini, "Mesenchymal stem cell secreted vesicles provide novel opportunities in (stem) cell-free therapy," Frontiers in Physiology, vol. 3, p. 359, 2012.

[17] R. C. Lai, F. Arslan, M. M. Lee et al., "Exosome secreted by MSC reduces myocardial ischemia/reperfusion injury," Stem Cell Research, vol. 4, no. 3, pp. 214-222, 2010.

[18] Y. Qin, R. Sun, C. Wu, L. Wang, and C. Zhang, "Exosome: a novel approach to stimulate bone regeneration through 
regulation of osteogenesis and angiogenesis," International Journal of Molecular Sciences, vol. 17, no. 12, 2016.

[19] L. Hu, J. Wang, X. Zhou et al., "Exosomes derived from human adipose mesenchymal stem cells accelerates cutaneous wound healing via optimizing the characteristics of fibroblasts," Scientific Reports, vol. 6, no. 1, article 32993, 2016.

[20] C. Li, A. Harper, J. Puddick, W. Wang, and C. McMahon, "Proteomes and signalling pathways of antler stem cells," PLoS One, vol. 7, no. 1, article e30026, 2012.

[21] M. Cegielski, I. Izykowska, M. Chmielewska et al., "Characteristics of MIC-1 antlerogenic stem cells and their effect on hair growth in rabbits," In Vivo, vol. 27, no. 1, pp. 97-106, 2013.

[22] C. Li, "Deer antler regeneration: a stem cell-based epimorphic process," Birth Defects Research. Part C, Embryo Today, vol. 96, no. 1, pp. 51-62, 2012.

[23] U. Kierdorf, C. Li, and J. S. Price, "Improbable appendages: deer antler renewal as a unique case of mammalian regeneration," Seminars in Cell \& Developmental Biology, vol. 20, no. 5, pp. 535-542, 2009.

[24] C. Li, F. Yang, and A. Sheppard, "Adult stem cells and mammalian epimorphic regeneration-insights from studying annual renewal of deer antlers," Current Stem Cell Research \& Therapy, vol. 4, no. 3, pp. 237-251, 2009.

[25] Z. F. Lu, S. Q. Cai, J. J. Wu, and M. Zheng, "Biological characterization of cultured dermal papilla cells and hair follicle regeneration in vitro and in vivo," Chinese Medical Journal, vol. 119, no. 4, pp. 275-281, 2006.

[26] P. Zhang, R. E. Kling, S. K. Ravuri et al., "A review of adipocyte lineage cells and dermal papilla cells in hair follicle regeneration," Journal of Tissue Engineering, vol. 5, article 2041731414556850, 2014.

[27] M. E. Balana, H. E. Charreau, and G. J. Leiros, "Epidermal stem cells and skin tissue engineering in hair follicle regeneration," World Journal of Stem Cells., vol. 7, no. 4, pp. 711-727, 2015.

[28] J. Kishimoto, R. E. Burgeson, and B. A. Morgan, "Wnt signaling maintains the hair-inducing activity of the dermal papilla," Genes \& Development., vol. 14, no. 10, pp. 1181-1185, 2000.

[29] K. S. Houschyar, A. Momeni, M. N. Pyles, Z. N. Maan, A. J. Whittam, and F. Siemers, "Wnt signaling induces epithelial differentiation during cutaneous wound healing," Organogenesis, vol. 11, no. 3, pp. 95-104, 2015.

[30] J. L. Whyte, A. A. Smith, and J. A. Helms, "Wnt signaling and injury repair," Cold Spring Harbor Perspectives in Biology, vol. 4, article a008078, 2012.

[31] S. S. Cheon, Q. Wei, A. Gurung et al., "Beta-catenin regulates wound size and mediates the effect of TGF-beta in cutaneous healing," The FASEB Journal, vol. 20, no. 6, pp. 692-701, 2006.

[32] H. Guo, Y. Xing, Y. Liu et al., "Wnt/ $\beta$-catenin signaling pathway activates melanocyte stem cells in vitro and in vivo," Journal of Dermatological Science, vol. 83, no. 1, pp. 45-51, 2016.

[33] T. Andl, S. T. Reddy, T. Gaddapara, and S. E. Millar, "WNT signals are required for the initiation of hair follicle development," Developmental Cell, vol. 2, no. 5, pp. 643-653, 2002.

[34] A. B. Mohd Hilmi and A. S. Halim, "Vital roles of stem cells and biomaterials in skin tissue engineering," World Journal of Stem Cells., vol. 7, no. 2, pp. 428-436, 2015.

[35] S. J. Forbes and N. Rosenthal, "Preparing the ground for tissue regeneration: from mechanism to therapy," Nature Medicine, vol. 20, no. 8, pp. 857-869, 2014.

[36] J. Morhayim, R. Rudjito, J. P. van Leeuwen, and M. van Driel, "Paracrine signaling by extracellular vesicles via osteoblasts,"
Current Molecular Biology Reports, vol. 2, no. 1, pp. 48-55, 2016.

[37] M. Nawaz, F. Fatima, K. C. Vallabhaneni et al., "Extracellular vesicles: evolving factors in stem cell biology," Stem Cells International, vol. 2016, pp. 1-17, 2016.

[38] K. M. Cadigan and M. L. Waterman, "TCF/LEFs and Wnt signaling in the nucleus," Cold Spring Harbor Perspectives in Biology, vol. 4, no. 11, 2012.

[39] E. M. Green and R. T. Lee, "Proteins and small molecules for cellular regenerative medicine," Physiological Reviews, vol. 93, no. 1, pp. 311-325, 2013.

[40] S. Bollini, C. Gentili, R. Tasso, and R. Cancedda, "The regenerative role of the fetal and adult stem cell secretome," Journal of Clinical Medicine., vol. 2, no. 4, pp. 302-327, 2013.

[41] L. Timmers, S. K. Lim, F. Arslan et al., "Reduction of myocardial infarct size by human mesenchymal stem cell conditioned medium," Stem Cell Research, vol. 1, no. 2, pp. 129-137, 2007.

[42] D. G. Phinney and M. F. Pittenger, "Concise review: MSCderived exosomes for cell-free therapy," Stem Cells, vol. 35, no. 4, pp. 851-858, 2017.

[43] S. Bian, L. Zhang, L. Duan, X. Wang, Y. Min, and H. Yu, "Extracellular vesicles derived from human bone marrow mesenchymal stem cells promote angiogenesis in a rat myocardial infarction model," J Mol Med (Berl)., vol. 92, no. 4, pp. 387397, 2014.

[44] H. Xin, Y. Li, Y. Cui, J. J. Yang, Z. G. Zhang, and M. Chopp, "Systemic administration of exosomes released from mesenchymal stromal cells promote functional recovery and neurovascular plasticity after stroke in rats," Journal of Cerebral Blood Flow and Metabolism, vol. 33, no. 11, pp. 1711-1715, 2013.

[45] J. C. Gross, V. Chaudhary, K. Bartscherer, and M. Boutros, "Active Wnt proteins are secreted on exosomes," Nature Cell Biology, vol. 14, no. 10, pp. 1036-1045, 2012.

[46] B. Zhang, M. Wang, A. Gong et al., "HucMSC-exosome mediated-Wnt4 signaling is required for cutaneous wound healing," Stem Cells, vol. 33, no. 7, pp. 2158-2168, 2015. 


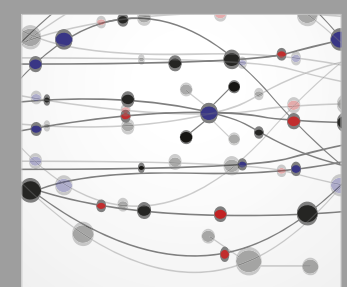

The Scientific World Journal
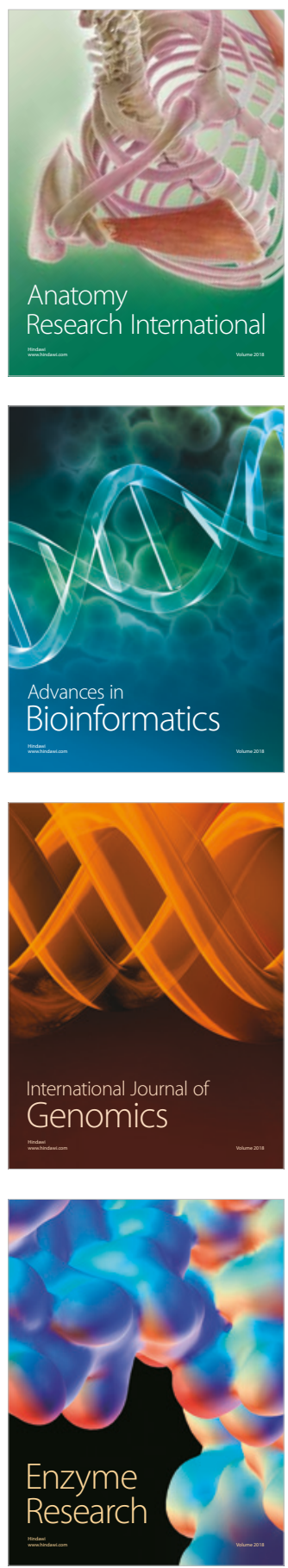
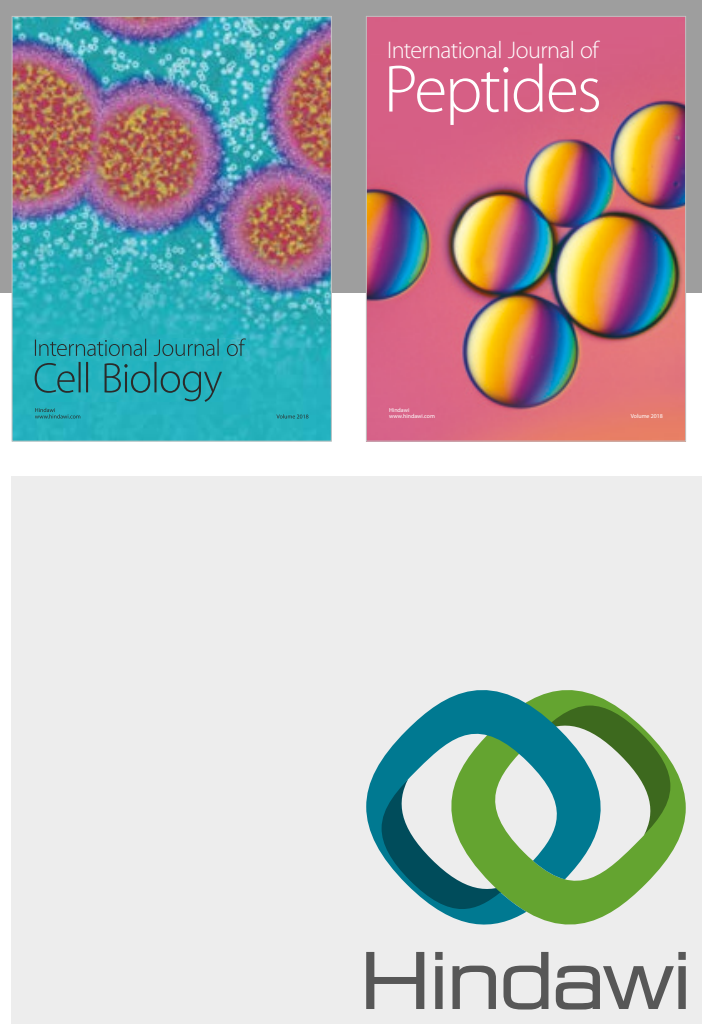

Submit your manuscripts at

www.hindawi.com
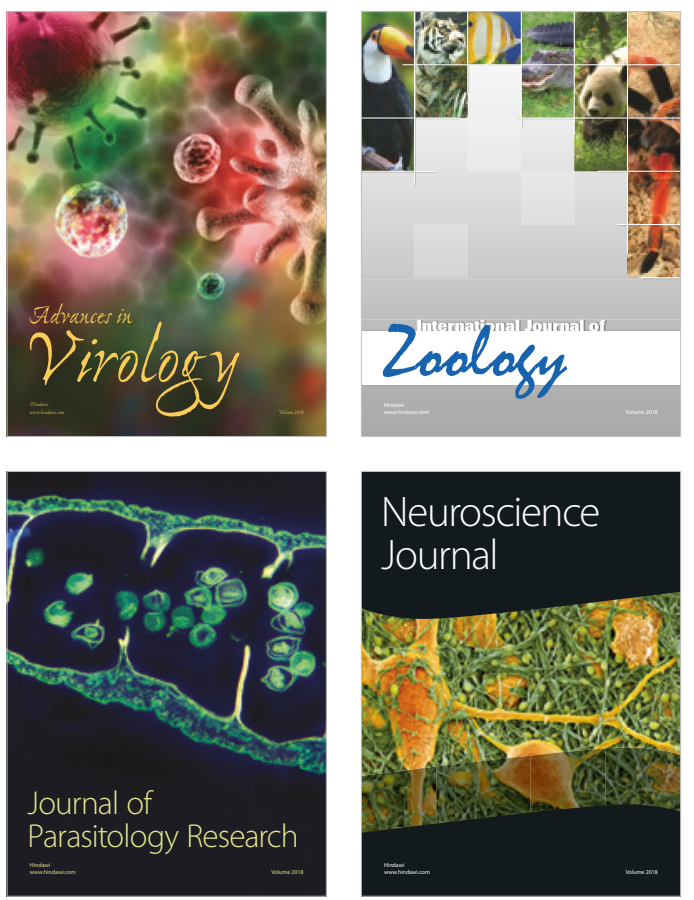
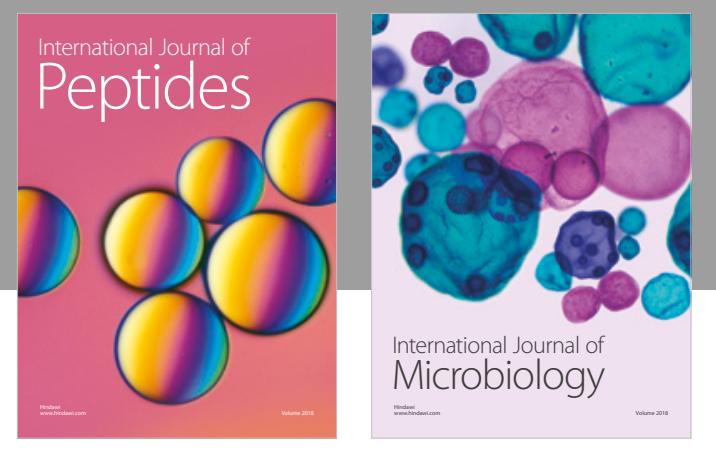

nternational Journal of Microbiology
Journal of
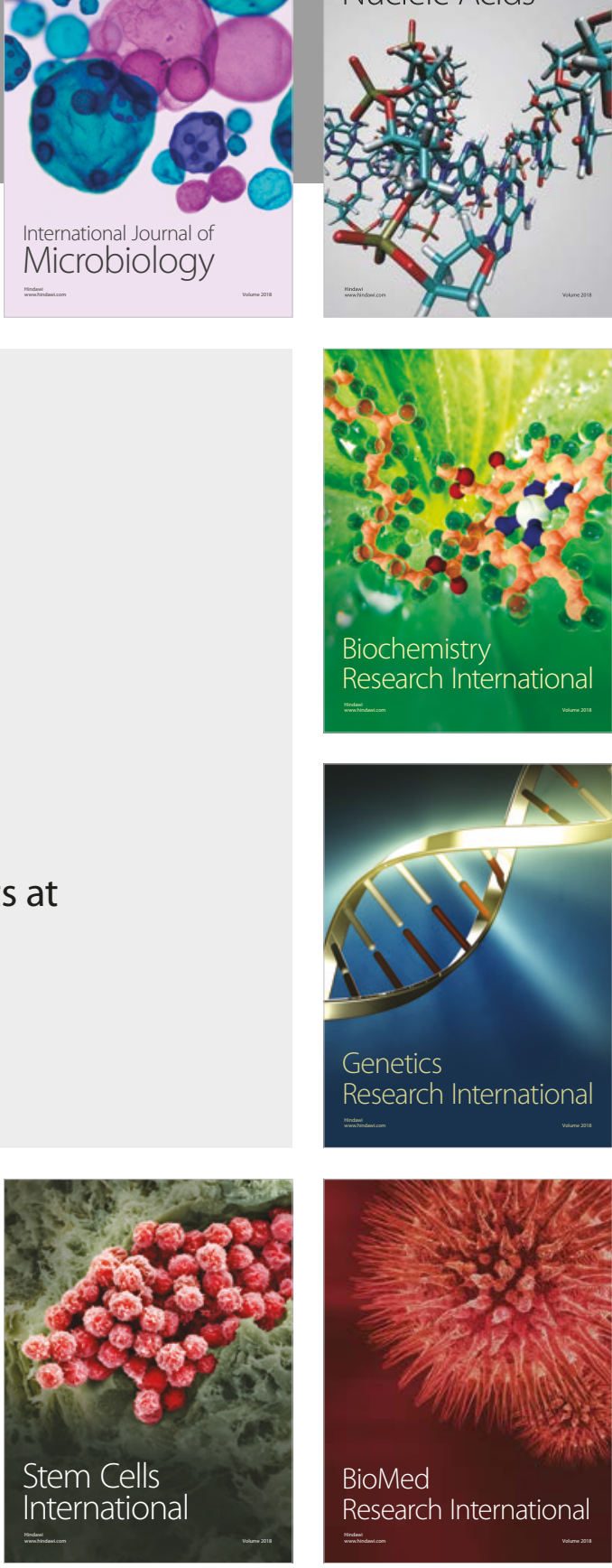
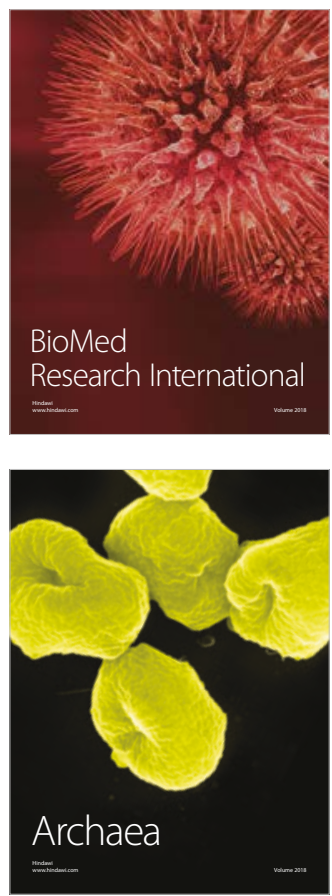